\title{
Glucose metabolism in normal aging and Alzheimer's disease: methodological and physiological considerations for PET studies
}

\author{
Lisa Mosconi
}

Received: 5 March 2013/Accepted: 25 June 2013/Published online: 10 July 2013

(C) Italian Association of Nuclear Medicine and Molecular Imaging 2013

\begin{abstract}
Alzheimer's disease (AD) is an age-dependent neurodegenerative disorder associated with progressive loss of cognitive function. 2-[ $\left.{ }^{18} \mathrm{~F}\right]$ fluoro-2-deoxy-D-glucose (FDG) positron emission tomography (PET) has long been used to measure resting-state cerebral metabolic rates of glucose, a proxy for neuronal activity. Several FDG PET studies have shown that metabolic reductions occur decades before onset of $\mathrm{AD}$ symptoms, suggesting that metabolic deficits may be an upstream event in at least some late-onset $\mathrm{AD}$ cases. This review explores this possibility, initially discussing the link between $\mathrm{AD}$ pathology, neurodegeneration, oxidative stress, and $\mathrm{AD}$, and then discussing findings of FDG PET hypometabolism in AD patients as well as in at-risk individuals, especially those with a first-degree family history of late-onset $\mathrm{AD}$. While the rare early-onset form of $\mathrm{AD}$ is due to autosomal dominant genetic mutations, the etiology and pathophysiology of age-dependent, late-onset $\mathrm{AD}$ is more complex. Recent FDG PET studies have shown that adult children of AD-affected mothers are more likely than those with AD-fathers to show AD-like brain changes. Given the connection between glucose metabolism and mitochondria, and the fact that mitochondrial DNA is maternally inherited in humans, it is here argued that altered bioenergetics may be an upstream event in those with a maternal history of late-onset AD. Biomarkers of $\mathrm{AD}$ have great potential for identifying $\mathrm{AD}$ endophenotypes in at-risk individuals, which may help direct investigation of potential susceptibility genes.
\end{abstract}

L. Mosconi $(\bowtie)$

Department of Psychiatry, Center for Brain Health, NYU School

of Medicine, 145 East 32nd Street, 2nd floor, New York,

NY 10016, USA

e-mail: Lisa.Mosconi@nyumc.org
Keywords Positron emission tomography $\cdot$ Cerebral metabolic rate of glucose (CMRglc) - Amyloid-beta . Preclinical detection $\cdot$ Normal aging $\cdot$ Maternal transmission

\section{Introduction}

Alzheimer's disease (AD), the leading cause of dementia in the elderly, is a neurodegenerative disorder characterized by an insidious onset and progressive declines in memory, attention, and language. At present, $\mathrm{AD}$ affects approximately $10 \%$ of individuals 65 years of age, with the prevalence doubling every 5 years up to age 80 , above which the prevalence exceeds $40 \%$ [1].

Longitudinal studies of normal individuals who go on to develop AD show that there is a somewhat abrupt transition in cognitive symptom decline between the preclinical stage and the early stage of AD. Gradual cognitive decline in the preclinical stage reaches an inflection point that gives way to a comparatively steep loss of cognitive abilities, which is the hallmark of clinical AD. Typically, the relatively rapid loss of cognitive abilities is what leads family members or caregivers to bring patients in for evaluation. By the time patients come in for diagnosis, too much irreversible brain damage may already have occurred for any treatments to be effective. Interventions, once developed, ideally would be implemented long before symptoms occur. While risk factors such as apolipoprotein E (ApoE) $\varepsilon 4$ genotype and family history have been identified, their predictive value remains to be established, and their presence may not be enough to justify the potential risks of medical interventions (as they become available) in non-symptomatic individuals. Therefore, another major goal in $\mathrm{AD}$ research is the identification of 
diagnostic markers, especially for the preclinical stages of disease when symptoms are not yet clinically relevant, such as in at-risk individuals.

Currently, a definite diagnosis of AD can only be made by neuropathology, which is regarded as the gold standard, and is based on post-mortem detection of specific pathological lesions: amyloid-beta $(A B)$ plaques in the extracellular space and blood vessels, intracellular neurofibrillary tangles (NFT), and neuronal and synaptic loss in specific brain regions [2-4]. Changes in brain histopathology, and consequently in brain structure and function, are known to precede the signs and symptoms of the disease by many years. AD pathology and associated neurodegeneration are estimated to begin 20-30 years before any clinical manifestations of the disease become evident [2-4]. Neurodegeneration is the umbrella term for the progressive loss of structure or function of neurons, including death of neurons. Many neurodegenerative diseases, including $\mathrm{AD}$, occur as a result of neurodegenerative processes. Plaques and tangles have long been considered upstream events in $\mathrm{AD}$, leading to subsequent neurodegeneration and neuronal apoptosis. According to a popular theoretical model in $\mathrm{AD}$, the "amyloid cascade hypothesis", A $\beta$ plaques increase during the preclinical phase of $\mathrm{AD}$, causing synapse loss and neuronal death [5]. However, other mechanisms such as oxidative stress are known to boost neurodegeneration, and may precede and promote $A B$ plaque deposition [6], thus having as strong an impact as pathological lesions. The central role for $A B$ in $A D$ is strongly supported by studies of the rare early-onset $(<60$ years) forms of familial $\mathrm{AD}$, which are caused by mutations in the amyloid precursor protein (APP), presenilin 1 and presenilin 2 genes, and are found in large multigenerational families with an autosomal dominant pattern of disease inheritance. These aspects have been discussed in countless publications (see, among others, [5, 7-10]). However, it is less clear whether $A B$ dysmetabolism is an upstream event also for the more common, lateonset form of $\mathrm{AD}$ (LOAD), which accounts for almost $99 \%$ of all AD cases. This review explores the possibility that oxidative stress and resulting glucose dysmetabolism are major key players in at least some LOAD cases, while not discounting the role of $\mathrm{A} \beta$ and tau pathology [11-16]. For more information about the genetics of $\mathrm{AD}$, including genetic risk factors for LOAD such as ApoE genotype, as well as a review of early-onset AD studies, please see the chapter by Nacmias et al. in this issue.

Some decades ago the widely accepted paradigm was that people with $\mathrm{AD}$ pathology had dementia and people without AD pathology did not. This simple division started blurring when pathology studies revealed plaques and tangles in a sizable fraction of elders who had died with their cognition intact, and that the number, density, and location of amyloid plaques were not particularly correlated with either symptoms or severity of $\mathrm{AD}[4,17]$. Biomarker and longitudinal aging studies of the past 20 years swept aside this old binary view in favor of a more complex and dynamic picture. At present, the prevailing view is that both pathological and clinical changes occur gradually over time, and that while there can be no clinical symptoms without $\mathrm{AD}$ pathology, there may be $\mathrm{AD}$ pathology without clinical symptoms. The presence of neurodegeneration, rather than plaques and tangles, is best associated with the onset of cognitive deficits in $\mathrm{AD}$ [18]. It has become increasingly clear that $\mathrm{AD}$ is a polygenic and multifactorial disease, which may include different pathogenetic mechanisms that nonetheless result in similar clinical outcomes. The following paragraphs explore this possibility, initially discussing the link between $\mathrm{AD}$ pathology and neurodegeneration, and focusing on the metabolic pathways involved in neuronal function and bioenergetics, and then discussing the relationship between glucose metabolism, oxidative stress and AD. There will then follow a summary of positron emission tomography (PET) imaging of brain glucose metabolism in AD and a discussion of recent findings of selective reductions of PET brain glucose metabolism in adult children of mothers, but not fathers, affected by LOAD. These data led to the discovery of a maternally inherited AD endophenotype characterized by progressive brain hypometabolism, increased amyloid load, atrophy, and oxidative stress. Finally, given the connection between glucose metabolism and mitochondria, and the fact that mitochondrial DNA (mtDNA) is maternally inherited in humans, it is argued that altered bioenergetics may be an upstream event in individuals with a maternal family history of $\mathrm{AD}$.

\section{Alzheimer's disease and bioenergetics}

Highly metabolic neurons are affected early in Alzheimer's disease

The brain regions experiencing the earliest changes in $\mathrm{AD}$ are the medial temporal lobe (MTL) structures, entorhinal and perirhinal cortex, and hippocampus [2, 3, 19]. In the neocortex, the pyramidal cells anatomically connected to the entorhinal cortex and the CA1 and subiculum regions of the hippocampus are particularly prone to NFT formation and degeneration, whereas primary sensorimotor and occipital areas and the cerebellum exhibit minimal pathology and neuronal loss [2, 3, 19]. Disruption of the pyramidal neurons in the perforant path is thought to disconnect the hippocampus from the rest of the cortex, strongly contributing to the decline in memory observed in early AD [18]. Despite a predilection for the neocortex 
early on, $A ß$ depositions are also found in the MTL at later stages of disease [19]. Soluble A $\beta$ oligomers disrupt longterm potentiation and reduce synaptic plasticity in the hippocampus early in the course of the disease [5]. The medial temporal regions and functionally connected neocortex are most affected in $\mathrm{AD}$, but the pathology is not uniform, nor does it affect all cell types. Despite recent advances in our understanding of the molecular basis and pathophysiology of neurodegenerative disorders, the problem of selective neuronal vulnerability has proved difficult to solve. Why is the hippocampus initially affected in AD? Why and how does neuronal death spread to other brain regions as the disease progresses?

Recent advances have begun to show how cellular and molecular changes that occur during normal aging render neurons vulnerable to degeneration, and how disease-specific genetic and environmental factors determine which neurons succumb and which are resistant to advancing pathology. The physical and molecular characteristics of neurons, their functional properties, and their location in neural circuits are all likely to influence their fate during aging (for reviews, see $[13,18]$ ). Large projection neurons with relatively long axons are most damaged in AD. These neurons have three main characteristics:

1. They have high energy requirements. These neurons have high metabolic rates. As such, their functionality is directly dependent on glucose availability and utilization.

2. They rely primarily on axonal transport for functional support (anterograde and retrograde). The axons of cortico-cortical projection neurons travel long distances, which makes these neurons more prone to receiving multiple insults and more sensitive to cytoskeletal dysfunction [13, 18].

3. They have a large cell-surface area. This increases exposure of the cells to toxic environmental conditions.

Normal synapse function requires a multitude of coordinated mechanisms, including generation of gene products responsible for the formation and maintenance of membrane complexes; synthesis and delivery of mRNAs, proteins, and transmitters; regulation of vesicle trafficking; release and reuptake; and many more. It is implied that, for all these actions to be performed efficiently, sufficient energy substrates must be supplied and utilized. Under normal physiological conditions, the brain relies almost exclusively on glucose as its main substrate for energy production. As there are only minor glycogen stores in the brain, a permanent supply of glucose from the blood stream is necessary to maintain brain function $[20,21]$. In the brain, the free energy necessary to drive most cellular reactions is derived from phosphorylation of ATP, which is mostly produced in the mitochondria from the oxidation of glucose under aerobic conditions. Interruptions of the glucose supply cause almost immediate failure of brain function, as can be seen from the rapid loss of consciousness that follows interruption of blood supply to the brain, as due to severe hypoglycemia [22]. Disruptions in glucose metabolism are closely associated with synaptic dysfunction [20, 21]. In keeping with this observation, recent evidence suggests that altered neuronal energy metabolism is a very early change, and a strong correlate of clinical impairment in $\mathrm{AD}$. It is here hypothesized that a deficit in the utilization of glucose, rather than reduced glucose availability, is involved in some LOAD cases, as reviewed below.

\section{Brain glucose metabolism}

\section{Molecular basis of glucose metabolism}

Brain glucose metabolism is comprised of a series of processes by which glucose is converted into ATP to be used for cellular energy: glycolysis in the cytoplasm, Krebs cycle and oxidative phosphorylation in the mitochondria. As shown in Fig. 1, under aerobic conditions, glucose is transported, by glucose transporters, from blood to tissue where it is phosphorylated into glucose-6-phosphate (G-6$\mathrm{P})$ by the hexokinase and glucokinase enzymes; it is then further metabolized down the glycolytic pathway until it is reduced to pyruvate. Pyruvate then enters the mitochondria to undergo further enzymatic degradation under oxidative phosphorylation (OXPHOS). OXPHOS is the process that accounts for the high ATP yield by the cells, and it is essential to the maintenance of cellular homeostasis and function. Pyruvate combines with oxygen to form an acetyl group, releasing carbon dioxide. The acetyl group $\left(\mathrm{CH}_{3} \mathrm{CO}\right)$ is then combined with coenzyme $\mathrm{A}$ as acetyl coenzyme A, and enters the Krebs cycle. During this series of reactions, each acetyl group is oxidized to form two molecules of carbon dioxide, and the energy released is transferred to four electron carrier molecules that form the electron transport chain (ETC, i.e. mitochondrial respiration). The ETC and OXPHOS are two linked processes, occurring in the mitochondrial inner membrane (IMM), that allow the mitochondria to reoxidize NADH to $\mathrm{NAD}^{+}$ and $\mathrm{FADH}_{2}$ to FAD and produce ATP. Four membranebound complexes embedded in the IMM and electrically connected by electron carriers are involved in the ETC. These complexes are NADH dehydrogenase (Complex I), succinate dehydrogenase (Complex II), cytochrome bc1 complex (Complex III), and cytochrome $c$ oxidase (COX, Complex IV). Complexes I, III, and IV are proton pumps that, while transferring electrons down the ETC, move $\mathrm{H}^{+}$ across the IMM against the concentration gradient, 


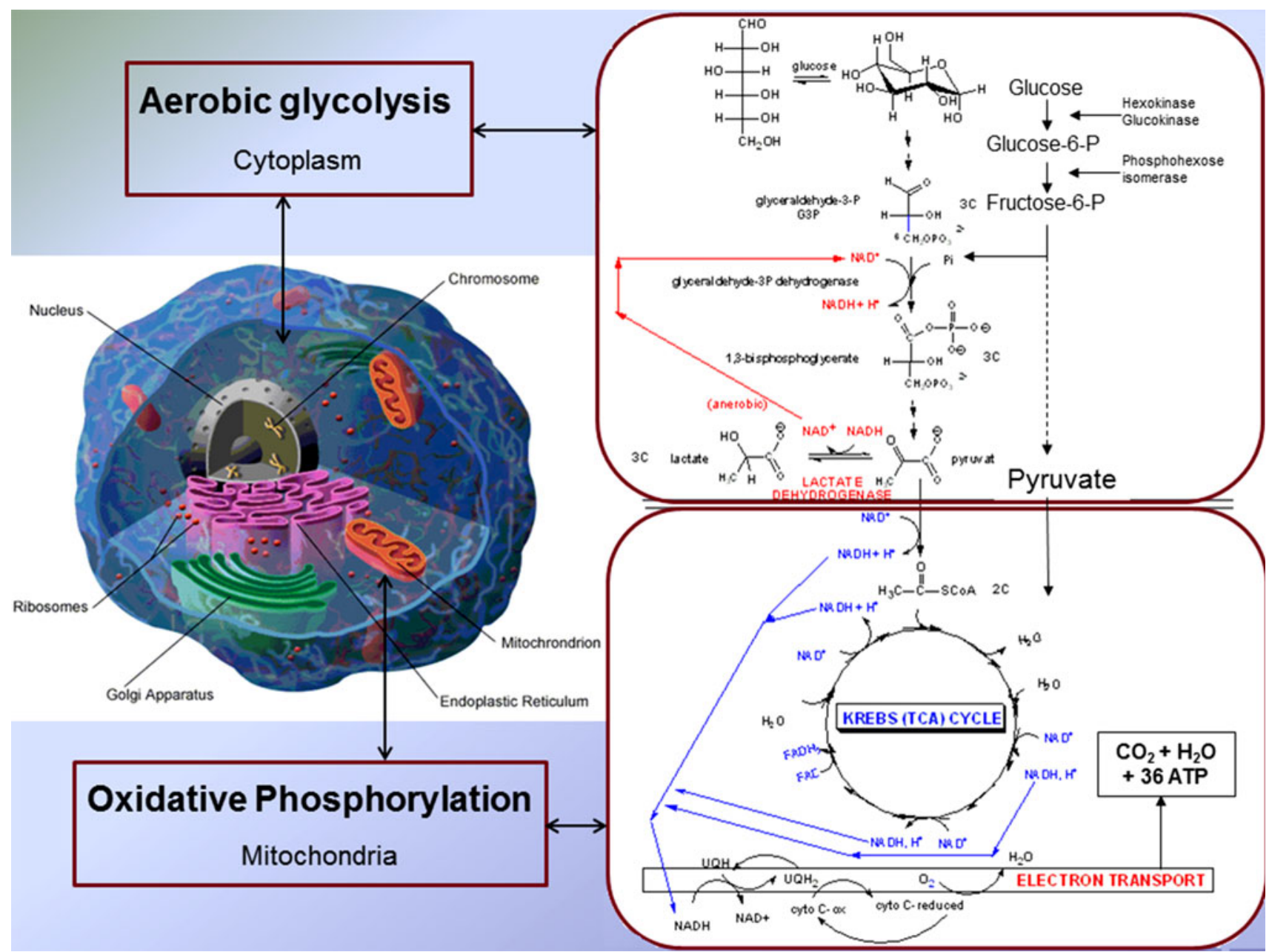

Fig. 1 Aerobic cell respiration: glucose metabolism and the electron transport chain in mitochondria. During respiration, glucose is oxidized to pyruvate down the glycolytic pathway in the cytoplasm. Pyruvate enters the mitochondria and the Krebs cycle. Each time the fuel molecule is oxidized, the released electrons are collected by

inducing a proton gradient from the matrix to the intermembrane space. This proton gradient is used by $\mathrm{F}_{0} \mathrm{~F}_{1}$ ATP-synthase (Complex V of the ETC) to produce ATP via OXPHOS of ADP to ATP. The hydrolysis of ATP is subsequently used to drive almost every energy-requiring reaction in the cell.

Although electron transport occurs with great efficiency, a small percentage of electrons are prematurely leaked to oxygen at the ETC Complex I and III steps, resulting in the formation of reactive oxygen species (ROS), including free radicals and peroxides. Oxidative stress is caused by an imbalance between production of ROS and the cell's ability to detoxify them [15]. The generation of ROS, due to their reactions with macromolecules, can lead to immediate oxidative damage of cell lipids, proteins, and DNA, which eventually results in neuronal damage and death $[13,15,16]$. Therefore, reduced activity of one or more of the ETC enzymes could compromise ATP electron carriers and then delivered to the electron transport chain (ETC). The electrons are transferred from protein to protein down the ETC chain (oxidative phosphorylation), which results in production of $\mathrm{CO}_{2}$, water, and ATP: $\mathrm{C}_{6} \mathrm{H}_{12} \mathrm{O}_{6}+6 \mathrm{O}_{2}=>6 \mathrm{CO}_{2}+6 \mathrm{H}_{2} \mathrm{O}+$ 30ATP

synthesis and increase production of ROS, with damage to neurons. In particular, impaired Complex I and III functioning affects the activity of Complex IV (COX), the mitochondrial enzyme responsible for the activation of oxygen for aerobic energy metabolism [23]. COX is localized primarily in mitochondria near excitatory synapses in neuronal dendrites, and its activity is closely linked to the regulation of glucose metabolism in the synapses and the production of ATP in mitochondria [23]. Impaired COX activity can lead to both reduced oxidative metabolism and increased production of ROS.

Oxidative stress and altered glucose metabolism in Alzheimer's disease

Altered oxidative metabolism in AD was noted as far back as 1965 [24]. Since then, several studies have confirmed the presence of severe oxidative stress in brain tissue, blood 
platelets, and fibroblasts in AD. At post-mortem, there is evidence for extensive oxidative stress in $\mathrm{AD}$ brains, in which basically all cellular macromolecules (protein, DNA, lipids) are found in an oxidized form [25-27]. Oxidative stress is most prominent in the brain regions showing degeneration in AD, particularly in the form of reduced COX activity in the parieto-temporal, frontal and posterior cingulate cortices, and hippocampus [25-27]. Several in vivo studies of peripheral markers have shown that levels of ETC enzymes, particularly COX activity, are severely reduced in blood platelets and fibroblasts in $\mathrm{AD}$ and in patients with mild cognitive impairment (MCI), often a prodrome to AD, compared with controls [27-30].

Lipid membrane peroxidation is another early event following ROS increases; it derives from oxidative degradation of polyunsaturated fatty acids in cell membranes. Lipid peroxidation products of arachidonic acid and docosahexaenoic acid are most prominent in the hippocampus and association cortex in AD. Increased isoprostane levels, a marker of lipid membrane peroxidation, are found in brain regions vulnerable to NFT formation. Isoprostanes correlate with the extent of neurodegeneration in $\mathrm{AD}$, and increases in this biomarker precede $\mathrm{A} B$ deposition in transgenic animals [6].

Overall, these studies provide evidence for peripheral oxidative stress, such as in blood platelets and fibroblasts that are non-degenerating tissue and should not be affected by central nervous system (CNS) pathology. This suggests that COX reductions may not be simply a secondary, or epiphenomenal, consequence of neurodegeneration, but may instead be an expression of systemic damage in AD. These findings support an alternative to the amyloid cascade hypothesis that has long dominated AD research. It is plausible that mitochondria and cell bioenergetics regulate $\mathrm{A} \beta$, and changes in mitochondrial function and cell bioenergetics occur upstream to $A \beta$ changes in sporadic $A D$, at least in some cases [12]. From a mechanistic perspective, perturbed mitochondrial function may promote the processing of APP to amyloidogenic derivatives or AB itself by increasing free radical production [6, 31]. A deficient energy metabolism resulting from defective mitochondrial function and increased oxidative damage may change the overall oxidative microenvironment during progression of LOAD, disposing neurons to dysfunction and degeneration $[14,15]$. The relationship of AD to aging, a process closely related to various aspects of mitochondrial function and integrity, has been used to argue that mitochondria may constitute the apex of biochemical cascades in LOAD [11, 12]. The core assumptions of this model are that a person's genes determine baseline mitochondrial function and durability; this durability determines how mitochondria change with advancing age, and critical changes in mitochondrial function initiate other pathologies characteristic of AD. While placing mitochondria at the apex of an AD cascade remains controversial, it is increasingly recognized that oxidative stress plays an important role. Investigations of oxidative stress and brain glucose metabolism are particularly suited to test this hypothesis in vivo, particularly by means of neuroimaging techniques in combination with mitochondria biochemistry, proteomic, and genomic data.

\section{Positron emission tomography imaging of brain glucose metabolism}

The FDG signal: biochemistry and modeling

Imaging techniques are invaluable in diagnosing $\mathrm{AD}$, as they are generally non-invasive and relatively easy for patients to tolerate. Among these, PET has long been used in the diagnosis of neurodegenerative diseases. In particular, the development of 2-[ $\left.{ }^{18} \mathrm{~F}\right]$ fluoro-2-deoxy-D-glucose (FDG) in the late 1970s was a major factor in expanding the scope of PET imaging [32]. The FDG tracer is an analog of glucose that couples fluorine-18 $\left({ }^{18} \mathrm{~F}\right)$ (half-life: $110 \mathrm{~min}$ ) to glucose. Metabolically active tissues within the body, such as tumors, the brain and heart, are easily visualized with FDG as they take up more glucose than other tissues. The concentrations of imaged tracer reflect tissue metabolic activity, in terms of regional glucose uptake. FDG PET offers the unique capability to both visualize and quantitate the resting-state cerebral metabolic rate of glucose (CMRglc), which is a proxy for neuronal activity [20,33] and a direct index of synaptic function and density [34, 35]. The FDG PET method was developed on the basis of Sokoloff's landmark autoradiographic studies over 25 years ago $[32,33,36]$ and was validated for the human brain by Michael Phelps in 1979 [37]. PET researchers are often asked by molecular and cell biologists what exactly is reflected in the FDG signal. This is a very important question, as the term "glucose metabolism", in nuclear medicine and biology, is used to describe rather different substrates. In biology, glucose metabolism is the process by which the carbohydrate is broken down via multiple enzymatic pathways to produce ATP during oxidative phosphorylation, or lactate during anaerobic metabolism. FDG shares a similar beginning, but not the same fate.

After image acquisition, correction for attenuation and scatter, and reconstruction, the FDG PET signal is modeled either using absolute quantitation or by means of 'relative' measurements. As a detailed review of PET kinetic modeling would be beyond the scope of this review, only the essential facts are discussed in what follows, while the reader is referred to the article by Berti et al. in this issue. Absolute quantitation of CMRglc is based on measurement 
of the accumulation of phosphorylated 2-deoxyglucose in the brain relative to the rate of exposure (the input function) while taking into account the effect of the radiolabeled derivative. The method was first developed with carbon-14-labeled deoxyglucose $\left({ }^{14} \mathrm{C}-\mathrm{DG}\right)$ and was designed specifically to take advantage of the localization made possible by quantitative autoradiography [33, 38]. The deoxyglucose (DG) method was developed to measure the rates of glucose utilization simultaneously in all structural and functional compartments of the CNS in conscious animals [33]. The use of DG served to isolate the chemical process under study to a well-defined reaction: the hexokinase-catalyzed phosphorylation of hexose, which is the first step in the biochemical pathway of glucose metabolism. The method was subsequently adapted to human brain studies with PET, by labeling DG with ${ }^{18} \mathrm{~F}$, another gamma emitting isotope [32, 37]. The FDG PET method is based on the identification of three compartments involved in glucose metabolism (Fig. 2). Glucose is transported bidirectionally from blood to tissue by glucose transporters, mainly GLU1 (found in the blood brain barrier and on astrocytes) and GLU3 (the major nerve glucose transporter) at a specific rate $\left(K_{1}, \mathrm{~mL} / \mathrm{g} / \mathrm{min}\right)$. A small amount flows back into the bloodstream $\left(k_{2}, \min ^{-1}\right)$. After transfer to tissue, glucose is phosphorylated into G-6-P by the hexokinase enzyme $\left(k_{3}\right.$, i.e., phosphorylation rate; $\min ^{-1}$ ), and is then further metabolized down the glycolytic pathway to pyruvate. A small amount of G-6-P undergoes dephosphorylation ( $k_{4}$, dephosphorylation rate).

FDG is an ${ }^{18}$ F-labeled analog of glucose. Its molecular composition is similar to that of glucose except for a missing hydroxide at the second carbon position, which is replaced by ${ }^{18} \mathrm{~F}$ (Fig. 2). After a bolus injection into the blood, FDG is transported bidirectionally by the same carriers that transport glucose across the blood brain barrier, enters brain tissue, and is phosphorylated into FDG-6phosphate (FDG-6-P) by the hexokinase enzyme. However, because of the missing oxygen, the following enzyme, glucose-P-isomerase, does not recognize the molecule and FDG-6-P cannot be converted to fructose-6-phosphate (Fig. 2). FDG-6-P, once formed, remains essentially "trapped" in tissue. The three-compartment model with four rate constants is often simplified by assuming that the dephosphorylation rate of FDG-6-P in brain tissue is so low that it can be ignored $\left(k_{4}=0\right)$ over a standard acquisition time of $60 \mathrm{~min}$ post injection. Therefore, FDG PET measures of CMRglc are based on blood flow, glucose transport, and phosphorylation by the hexokinase enzyme, and as such are restricted to the initial steps in glycolysis, without taking into account any further catabolism down the glycolytic pathway. FDG PET studies are performed after a patient has fasted, so that the endogenous glucose level is relatively constant, which is why the method yields estimates of "steady-state" or "resting-state" CMRglc. Blood flow and glucose transport are also relatively stable under normal physiological conditions and, therefore have limited impact on CMRglc determination, which is mostly dependent on the phosphorylation rate. The net rate of glucose phosphorylation can be calculated by the operational equation:

$R_{\mathrm{i}}=\frac{C_{\mathrm{i}}^{*}(T)-K_{1}^{*} \int_{0}^{T} C_{\mathrm{p}}^{*}(t) \mathrm{e}^{-\left(k_{2}^{*}+k_{3}^{*}\right)(T-t)} \mathrm{d} t}{\frac{\lambda V_{\mathrm{m}}^{*} K_{\mathrm{m}}}{\Phi V_{\mathrm{m}}}\left(\int_{0}^{T} \frac{C_{\mathrm{p}}^{*}(t)}{C_{\mathrm{p}}} d \mathrm{t}-\int_{0}^{T} \frac{C_{\mathrm{p}}^{*}(t)}{C_{\mathrm{p}}} \mathrm{e}^{-\left(k_{2}^{*}+k_{3}^{*}\right)(T-t)} d \mathrm{t}\right),}$

where $R_{\mathrm{i}}$ is the metabolic rate of glucose, $C_{\mathrm{i}}{ }^{*}$ represents total concentration of ${ }^{18} \mathrm{~F}$ in a homogeneous tissue in the brain; $C_{\mathrm{p}}$ and $C_{\mathrm{p}}{ }^{*}$ are the ${ }^{18} \mathrm{~F}-\mathrm{FDG}$ and glucose concentrations in arterial plasma, respectively; $T$ is the duration of the experimental period following the pulse of tracer and $\mathrm{t}$ is the variable time; $K_{1} *$ and $k_{2} *$ are the rate constants for carrier-mediated transport of FDG from plasma to tissue and back again, respectively; $k_{3} *$ is the rate constant for phosphorylation of ${ }^{18}$ F-FDG by hexokinase; $\lambda$ equals the ratio of the distribution spaces of deoxyglucose and glucose in the tissue; $\Phi$ equals the fraction of glucose which, once phosphorylated, continues down the glycolytic pathway; $V_{\mathrm{m}}$ and $K_{\mathrm{m}}$ represent the maximal velocity and Michaelis-Menten constant of hexokinase for glucose, and $V_{\mathrm{m}} *$ and $K_{\mathrm{m}} *$ are the equivalent kinetic constants of hexokinase for ${ }^{18} \mathrm{~F}$-FDG.

In a simplified form, resting-state CMRglc is defined as the concentration of glucose in plasma (i.e., glycemia) divided by the lumped constant (LC), and multiplied by the metabolic rate of FDG $\left(K_{\mathrm{i}}\right)$ (Fig. 2). $K_{\mathrm{i}}$ is defined as the product of forward transport and phosphorylation rates $\left(K_{1} \times k_{3}\right)$ divided by the rate of backward transport plus the phosphorylation rate $\left(k_{2}+k_{3}\right)$. The LC accounts for the differences in transport and phosphorylation rates between D-glucose and 2-fluoro-2-deoxy-D-glucose, and is used to transform the FDG uptake rate to glucose uptake rate [33]. CMRglc is expressed as micromoles ( $\mu \mathrm{mol})$, or milligrams (mg), of glucose metabolized by $100 \mathrm{~g}$ of tissue per minute $\left(\mu \mathrm{mol} / 100 \mathrm{~g} \times \mathrm{min}^{-1}\right)$. While the above-mentioned operational equation remains the basis for all subsequent work in FDG modeling, substantial refinements to the method were achieved in later years [39-42]. For a detailed discussion on kinetic modeling, please see the article by Berti et al. in this issue.

In a steady state, the net rate through any step in a pathway equals the net rate through the overall pathway $[20,33,38]$. Therefore, by measuring the net rate of glucose phosphorylation in vivo, the FDG method yields the net rate of the entire glycolytic pathway in a steady state [20, 32, 33, 37, 38]. However, there is no information on whether glucose is eventually converted to ATP in mitochondria, turned into glycogen or lactate, or taken up in the pentose shunt. 


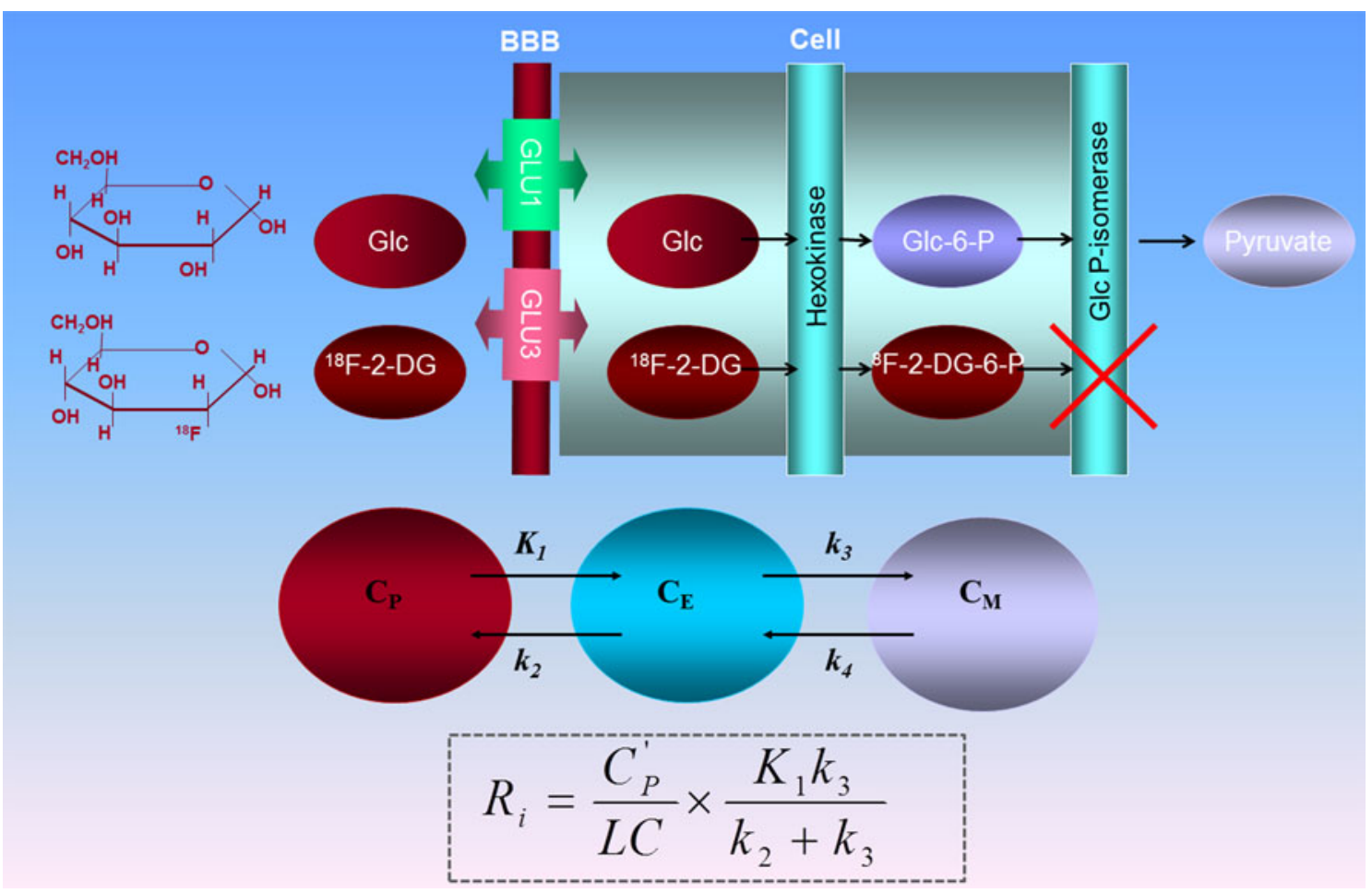

Fig. 2 Theoretical basis of FDG method of measuring local cerebral glucose utilization using a compartmental model. FDG and glucose (Glc) compete for the carriers that transport both between plasma and tissue (GLU1 and GLU3) and across the blood brain barrier (BBB), and for the hexokinase enzyme that phosphorylates them to their respective hexose 6-phosphates (Glc-6-P and $\left.{ }^{18} \mathrm{~F}-2 \mathrm{DG}-6-\mathrm{P}\right)$. The dashed arrow represents the possibility of glucose-6-phosphate (Glc6-P) hydrolysis by glucose-6-idromerase activity, in which case the molecule proceeds down the glycolytic pathway to pyruvate. ${ }^{18} \mathrm{~F}$ FDG-6-P is not further oxidized and remains trapped in tissue. In the theoretical model, $C_{\mathrm{P}}$ represents the total concentration of glucose in arterial plasma, $C_{\mathrm{E}}$ represents glucose concentration in the precursor tissue pool that serves as substrate for hexokinase, and $C_{\mathrm{M}}$ represents

\section{Absolute quantitation vs. simplified measurements}

Image data can be acquired in different ways and finding the best model to describe the tracer kinetics continues to be a lively area of research. The two principal FDG PET measurements are quantitative and non-quantitative (i.e., relative).

\section{Quantitative measurements}

These measurements are called 'quantitative' or 'absolute' as they are expressed as glucose metabolism units, i.e., micromoles of glucose taken up by $100 \mathrm{~g}$ of tissue per minute $(\mu \mathrm{mol} / 100 \mathrm{~g} / \mathrm{min})$. Quantitative measures can be derived using two main kinds of kinetic models, "compartmental" and "non-compartmental". Compartmental models describe the movement of the tracer between 'compartments' such as blood and the brain. The "fully glucose concentration in tissue. The constants $K_{1}, k_{2}$, and $k_{3}$ represent the rate constants for carrier-mediated transport of glucose from plasma to tissue, for carrier-mediated transport of glucose back from tissue to plasma, and for phosphorylation by hexokinase, respectively. The same model is applied to FDG. The metabolic rate of glucose $\left(R_{\mathrm{i}}\right)$ is estimated as a function of FDG concentration in plasma $\left(C_{\mathrm{P}}{ }^{\prime}\right)$, the lumped constant (LC), and the metabolic rate of FDG $\left[\left(K_{1} \times k_{3}\right) /\right.$ $\left.k_{2}+k_{3}\right)$ ]. While the three-compartment model includes four rate constants, it is often simplified by assuming that the dephosphorylation rate of FDG-6-phosphate in brain tissue $\left(k_{4}\right)$ is so low that it can be ignored $\left(k_{4}=0\right)$ over a standard acquisition time of $60 \mathrm{~min}$ post injection. $k_{4}$ was not, therefore, included in the equation at the bottom

dynamic" model is the "gold-standard" in PET studies and involves the simultaneous assessment of FDG kinetics in the brain and blood [37]. This is achieved via a dynamic acquisition of PET frames from the time of injection till 60 min post injection, and synchronized arterial plasma sampling throughout the time course of the study. Modeling is performed by plotting the time-activity curve of FDG concentration in brain and plasma to determine the rate at which the tracer is taken up by the brain and how rapidly it accumulates or clears, as reflected in the estimated kinetic rate constants $K_{1}, k_{2}$, and $k_{3}$. In contrast, "non-compartmental" kinetic models, also known as graphical analysis, generate estimates of the overall tracer metabolism instead of individual rate constants. Because of the invasive nature of arterial sampling, the complexity of analyzing metabolites in plasma, and the need to keep patients in the scanner for $60 \mathrm{~min}$ without moving, very few centers perform fully dynamic FDG PET studies in AD 
or in the elderly. The fully dynamic method has been used to develop and validate simplified FDG PET analysis methods that, while retaining the ability to yield quantitative CMRglc measures, overcome some of its limitations. Among these, the Gjedde-Patlak plot [43] and Sokoloff's autoradiographic model with population-based rate constants [33] yield quantitative CMRglc estimates while overcoming the need to acquire a full dataset.

\section{Non-quantitative measurements}

As an alternative to invasive kinetic modeling, simplified reference-tissue techniques have been developed to avoid blood sampling and to reduce scanning time, making the procedure more tolerable. These methods are helpful especially for large clinical trials and for patients with severe dementia. The most common among these methods, used particularly in oncology, is based on calculation of the standardized uptake value (SUV), a measurement of radioactive emissions localized to tissue normalized to the injected dose and patient body weight. Normalization of the PET signal to the injected dose can be readily performed on clinical PET scans to generate SUV images for visualization and clinical reading. To facilitate comparison over time and between subjects, a similar concept is employed by normalizing volumes or voxels of interest to a reference value, which is typically either whole-brain activity or activity of a region relatively unaffected by the disease process or drug intervention. This generates what is called a standardized uptake value ratio (SUVR), which is commonly used in clinical practice and also widely applied in research studies. Alternatively, for research studies, FDG count (i.e., signal intensity, $\mathrm{mCi}$ ) in a reference region is typically modeled as a continuous variable and controlled for as a covariate using linear regressions by means of proportional scaling. These measures are 'relative' in nature since metabolic activity in a target region is estimated relative to a reference region.

All these methods have advantages and disadvantages. Quantitative measures are invasive and computationally challenging, but are the closest to actual CMRglc. As such, they are particularly suitable for longitudinal studies and for characterizing the magnitude of CMRglc changes after drug interventions. When only localization of metabolic deficits is the main purpose of the study, e.g., localization of a tumor or of hypometabolic regions, quantification may be unnecessary $[44,45]$. Relative measurements are noninvasive and thus more easily applicable to clinical settings. However, relative measures are not linearly related to absolute measures and are, therefore, not proportional to CMRglc. The method is, therefore, highly dependent on the choice of reference region, which should not only remain metabolically stable over time but should also be unaffected by the disease process. This makes the method less reliable for severe dementia cases, for making comparisons between individuals, and for longitudinal examinations; indeed, relative measures, while helpful for visualization of hypometabolic regions, do not provide reliable information on the magnitude of the deficit. This method is instead particularly suitable for routine clinical PET interpretation of individual cases, especially thanks to the development of several well-validated, observer-independent, voxel-based methods. The most widely used FDG PET analysis methods for neurology research include, among others, Neurostat (University of Washington, Seattle) [46-50], the T-SUM tool implemented in PMOD [51], hypometabolic convergence indexes [52, 53], and Statistical Parametric Mapping procedures (SPM; Wellcome Department of Cognitive Neurology, London, UK) [54-57]. For a detailed review of technical and methodological factors involved in image analysis, please see the articles by Berti et al. and Herholz et al. in this issue. Overall, these techniques use image warping and spatial normalization procedures to enable voxel-based comparison of a patient's scans to a normative reference database, and yield statistical maps depicting deviations from norms at the individual voxel level, on a subject-by-subject basis. This facilitates detection of specific regional patterns of abnormalities in an automated and observer-independent fashion, thus overcoming some of the limitations of traditional visual inspection of scans. Voxel-based single-subject analysis yielded high sensitivity and specificity for the diagnosis of $\mathrm{AD}$ vs. controls and other dementias, as well as in the prediction of progression from $\mathrm{MCI}$ to $\mathrm{AD}$ dementia [46-57].

\section{What does the FDG PET signal represent at tissue level?}

As the resolution of modern PET scanners varies between 2 and $7 \mathrm{~mm}$, the technique does not provide information on metabolic activity at molecular level, but rather at tissue level. From a physiological standpoint, FDG PET is best suited to visualize changes in metabolism in anatomically and functionally defined regions, for several reasons. First, under normal physiological conditions, regional cerebral glucose utilization (rCMRglc) is coupled to regional cerebral blood flow (rCBF). Using a double-tracer autoradiographic technique with 2-deoxy-glucose to determine rCMRglc and radiolabeled iodoantipyrine to measure rCBF, several studies demonstrated a close relationship between the two measures in preclinical studies [21, 5860]. Similar results were obtained in humans using PET with oxygen-15-labeled water $\left(\mathrm{H}_{2} \mathrm{O}_{15}\right)$ for the measurement of $\mathrm{rCBF}[61,62]$. However, while the coupling 
between blood flow and glucose metabolism is likely to hold in neurodegeneration, $\mathrm{H}_{2} \mathrm{O}_{15}$ PET may not be as accurate as FDG PET for diagnosing AD [63].

Second, any increase in neuronal activity leads to an increase in $\mathrm{rCBF}$, and the adjustment of rCBF to changes in neuronal activity occurs within a few seconds [64, 65]. Changes in $\mathrm{rCBF}$ are thought to be neurogenically mediated vascular responses in preparation for an altered metabolic demand. While some authors reported that physiological stimulation was accompanied by increases in glucose uptake and blood flow without parallel increases in oxygen consumption [64, 66], there is general agreement about the relationship between rCMRglc, rCBF, and oxygen consumption at rest $[21,61,62,64,65]$. In the resting brain, oxygen is almost entirely used for oxidation of carbohydrates and functional metabolism (i.e., ATP production) with only a small fraction being needed for structural metabolism, including synthesis of glycogen in glial cells (2\%), nucleotides and lipids (5-10\%), and proteins $(0.5 \%)$ [21]. Therefore, under normal aerobic conditions, the glucose metabolism needed for ATP production increases proportionally to neuronal activity. Further evidence of a direct relationship between CMRglc and neuronal activity comes from studies that examined the effects of electrical stimulation on glucose consumption and showed an almost linear increase in glucose utilization with increasing frequency of stimulation [20, 21, 67, 68]. The opposite effect was also observed in sensory deprivation and pharmacological studies, in which decreases of functional activity led to reduced rates of glucose utilization [20, 58].

Third, a multitude of activation studies have shown that rCBF and rCMRglc increase in response to external stimulation in the brain regions responsible for the execution of a specific cognitive process. For example, rCBF and rCMRglc increase in the auditory cortex of individuals listening to music or sounds, and in the visual cortex after exposure to visual stimuli [69-72].

Finally, although it is widely accepted that CNS energy production is based almost exclusively upon the oxidation of glucose, there is a debate over exactly which cells, neurons or glia, are consuming the carbohydrate, and what work the energy is being used to support [35, 73, 74]. While glial cells outnumber neurons ten to one, most ATP is probably used by neurons to reverse ion fluxes associated with action potentials and excitatory postsynaptic currents. It has been estimated that as much as $70 \%$ of the energy derived from glucose oxidation is required to convert glutamate, the major neurotransmitter in the brain, to glutamine during glutamatergic transmission [35]. Therefore, the FDG PET signal mostly reflects excitatory neurotransmission. However, some studies have suggested that, during periods of elevated neuronal activity, only glia cells take up glucose and convert it to lactate via glycolysis. The lactate is then taken up by neurons and converted to pyruvate, which enters the citric acid cycle followed by oxidative phosphorylation [74]. Although this hypothesis remains to be confirmed, glial cell activity may account for up to $15 \%$ of oxidative cerebral glucose utilization. The link between glucose consumption and glutamate neurotransmission and recycling does not seem to be heavily dependent on the relative contributions of astrocytes and neurons to oxidative glucose consumption. In conclusion, glucose utilization as measured with FDG PET reflects mostly excitatory neuronal activity in brain tissue.

Overall, FDG PET images provide a visual and quantitative depiction of neuronal activity at tissue level and can be used to detect regional hypometabolism in disorders affecting brain function such as AD.

\section{FDG PET studies in the aging brain and Alzheimer's disease}

\section{Alzheimer's disease}

FDG PET studies have proved quite successful in diagnosing $\mathrm{AD}$. On FDG PET, AD is characterized by a specific regional pattern of CMRglc reductions in the parietotemporal cortex and posterior cingulate cortex (PCC) [49, 51, 75, 76] (Fig. 3). As the disease progresses, the frontal association cortices become involved, while the cerebellum, striatum, basal ganglia, and primary visual and sensorimotor cortices remain preserved $[75,77]$. This in vivo pattern of hypometabolism is found in the vast majority of clinically diagnosed AD patients and in over $85 \%$ of pathologically confirmed AD cases [76]. Despite some overlap, the characteristic $\mathrm{AD}$ pattern of CMRglc reductions yields high sensitivity in distinguishing $\mathrm{AD}$ from controls and from other neurodegenerative dementias [50, $51,76]$. The method yields high discrimination accuracy in patients with mild dementia as well as moderate-to-severe dementia [50, 51, 76]. Additionally, we and others have shown reduced metabolism and perfusion in the MTL (i.e., hippocampus, entorhinal and perirhinal cortex) of $\mathrm{AD}$ and MCI vs. controls [50, 78-83]. Findings of MTL hypometabolism are consistent with post-mortem and in vivo MRI observations that neuronal dysfunction begins in these regions, areas critically involved in the neural control of memory functions, and then spreads to the PCC, parietotemporal and frontal cortices later in the course of disease, in keeping with the progression of clinical symptoms [2, 3, $18,19]$. Studies have shown disconnection between histopathologically affected MTL regions and functionally associated areas, as well as reduced connectivity between the hippocampus and PCC/precuneus in normal (NL) 
(a)

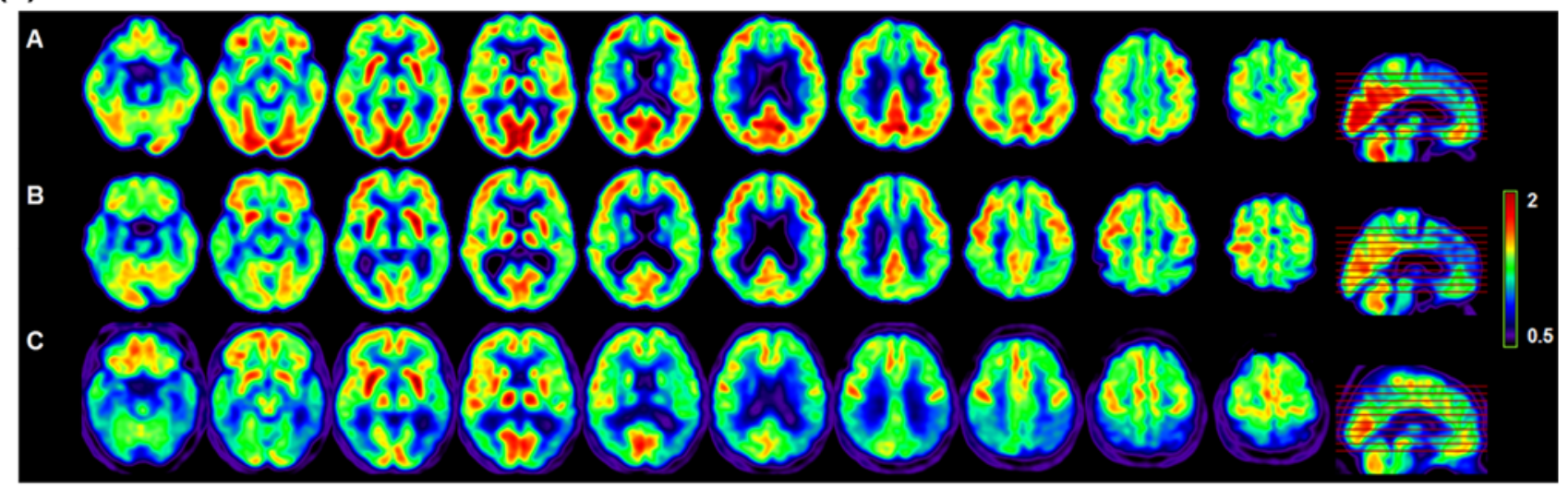

(b)

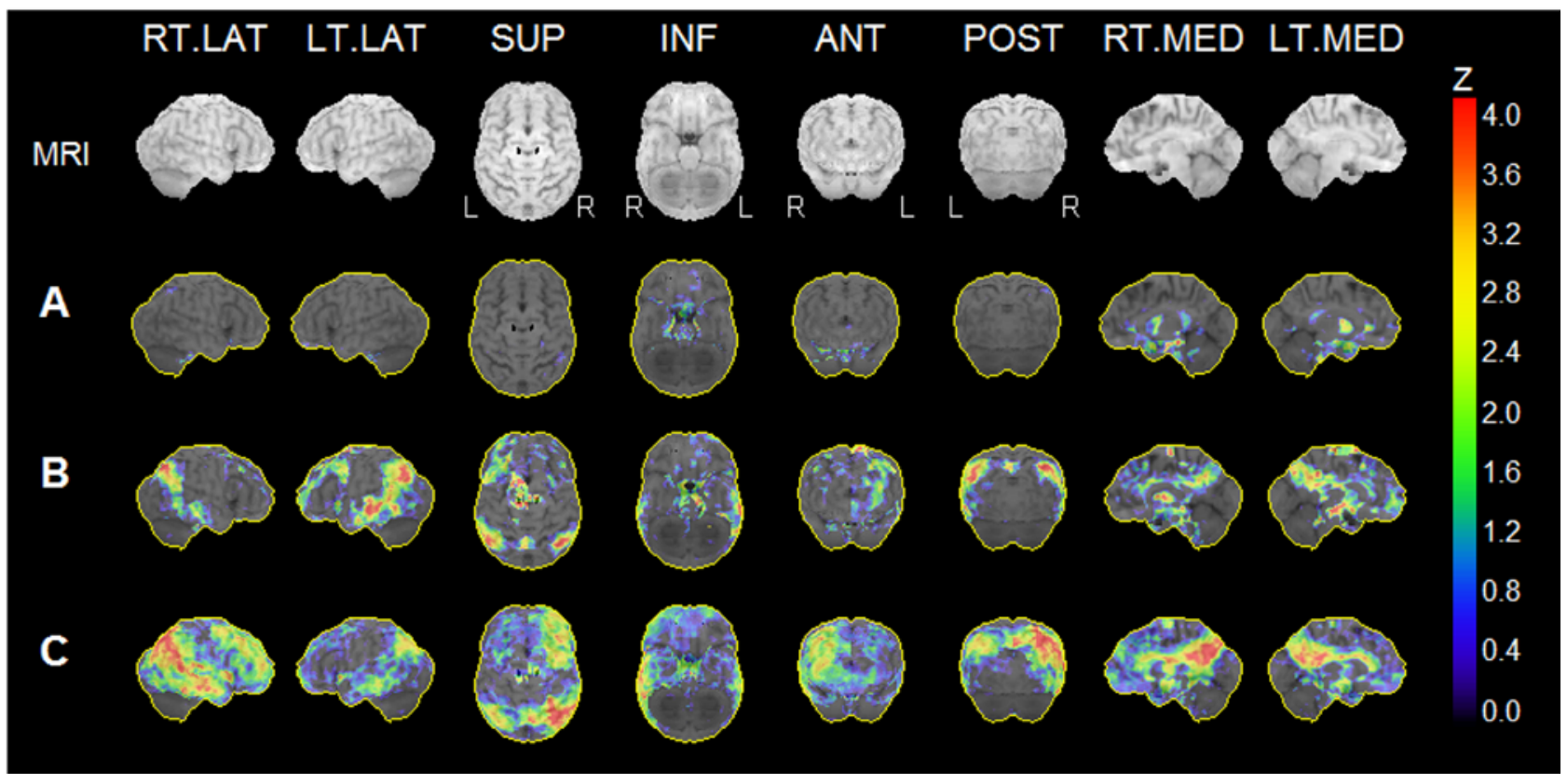

Fig. 3 FDG PET scans in normal aging and AD. a Three representative cases are shown: $A$ a 70 -year-old cognitively normal individual; $B$ a 70-year-old AD patient with mild dementia; $C$ a 75-year-old AD patient with severe AD. FDG PET scans are standardized uptake value ratios (SUVR) to pons (range 0.5-2). Regional hypometabolism is evident in the parieto-temporal regions of both $\mathrm{AD}$ patients compared with the control subject and is more pronounced in the severe $\mathrm{AD}$ than in the mild $\mathrm{AD}$ case. b 3D statistical surface

elderly harboring A $\beta$ burden [84-86]. While the MTL are not widely inspected in the FDG PET work up of AD dementia, these findings have implications for the pathophysiology of AD. A lack of MTL hypometabolism findings in AD might be due to biological, technical, and image analysis issues. It is possible that high synaptic density of temporo-parietal association cortex and PCC cortex would facilitate detection of glucose hypometabolism in these regions as compared with the MTL structures, which are rich in cell bodies but relatively poorer in terms of synaptic density [87]. For a discussion on the use of different image projection (SSP) maps and corresponding Z scores of the three representative cases shown in the (a) $(A-C)$. 3D-SSP maps showing reduced FDG uptake relative to a reference database are displayed on a color-coded scale ranging from 0 (black) to 4 (red). From left to right: 3D-SSP maps are shown on the right and left lateral, superior and inferior, anterior and posterior, right and left medial views of a standardized brain image. The pons was used as the reference region (color figure online)

analysis tools to detect hippocampal hypometabolism, please see, among other papers [75, 82, 87, 88].

With age, the brain undergoes both structural and functional alterations, probably resulting in reported cognitive declines. Relatively few investigations have sought to identify those areas that remain intact with aging, or undergo the least deterioration, and that might underlie cognitive preservation. In contrast to the AD-typical hypometabolic pattern, so-called normal aging has been associated with mild metabolic decreases in regions such as the lateral and medial frontal cortex, and anterior cingulate 
cortex, but not with posterior brain changes $[51,89,90]$. Across several studies, the most consistent finding in normal aging is decreased frontal metabolism, while temporal, parietal, and occipital lobe metabolism varies considerably among subjects within the same age group as well as over decades $[89,90]$. A large multicenter study directly comparing regional patterns of dementia-associated metabolic impairment and age-related decline in NL elderly showed that these patterns are quite distinct [51]. Even within the frontal cortex, normal aging had its largest effect in medial frontal regions, whereas AD-related changes occurred mostly in the lateral frontal cortex [51]. This suggests that $\mathrm{AD}$ is a disease process distinct from normal aging, with differences in regional patterns of glucose metabolism suggesting a substantial difference in the underlying pathophysiology [51]. Additionally, many neuropsychological studies have demonstrated age-related performance alterations in tests thought to reflect frontal and temporal lobe function, although there is little direct comparison of these hypothesized brain changes in vivo [91]. An FDG study of young (mean age 28 years) vs. late middle-aged to old (mean age 68 years) NL individuals showed age-related metabolic reductions in several frontal and temporal lobe regions [91]. The reductions ranged from 13 to $24 \%$, with the greatest changes occurring in the frontal lobes, especially the dorsal lateral frontal regions [91]. An elegant study comparing FDG and MRI in normal aging confirmed findings of predominant structural and functional deterioration of the frontal cortex, whereas the hippocampus, thalamus, and PCC were the least affected [92]. Relative preservation of the PCC and hippocampal regions was proposed as the parting of the ways between normal aging and $\mathrm{AD}$, as the latter is characterized by early and prominent deterioration of both structures [92].

An interesting finding is that clinical AD symptoms essentially never occur without CMRglc decreases, the extent of which is related to the severity of the cognitive impairment $[93,94]$. The AD pattern of glucose hypometabolism expands and worsens as clinical deterioration emerges. The hypometabolism that develops during the earliest stages in the MTL is concordant with the episodic memory deficits in AD that occur early in the disease [79, 80]. Expansion of hypometabolism to the parietal cortices has been associated with the visuospatial orientation deficits that contribute to debilitation [95]. Hypometabolism in the anterior cingulate occurs relatively early and has been correlated to the apathy that many patients experience [96, 97]. The deficits in the frontal cortex that become most notable later in the disease are consistent with the gradual loss of executive function [98, 99].

Due to its early sensitivity, FDG PET has been used to detect regional hypometabolism consistent with $\mathrm{AD}$ in non-demented individuals who would go on to develop
AD. A few longitudinal FDG PET studies of NL elderly subjects monitored the progression of some to $\mathrm{AD}$ and to MCI, often a prodrome to AD [100] compared with others who remained normal. These studies showed that CMRglc reductions in $\mathrm{AD}$-vulnerable regions precede the onset of dementia by many years [79, 80, 101-103] and predict decline from normal cognition to $\mathrm{MCI}$ and $\mathrm{AD}$ with over $80 \%$ accuracy $[79,80]$. In particular, PCC hypometabolism has consistently emerged as one of the main and earlier diagnostic findings in $\mathrm{AD}$, and it also accurately predicts conversion from MCI to AD [46, 49, 76, 104106]. Across AD patients, metabolic impairments seem to be more narrowly and consistently localized in the PCC/ precuneus than in the more extended frontal and temporoparietal association cortex, which could be the reason why maximum reduction in early $\mathrm{AD}$ is found there by parametric mapping [48, 49, 51]. It is worth noticing that the PCC is known to undergo early oxidative stress in AD, as reflected in a decrement of cytochrome oxidase, with progressive reduction within the superficial laminas linked to disease duration [107]. A recent genome-wide transcriptomic study showed that PCC hypometabolism on FDG PET may be associated with reduced neuronal expression of nuclear genes encoding subunits of the mitochondrial ETC [108]. Progressive CMRglc reductions in the PCC and other AD-vulnerable regions were observed years before clinical symptoms in a clinico-pathological series of subjects followed with longitudinal in vivo FDG PET scans from normal cognition to clinical diagnosis and post-mortem confirmation of AD [102]. Additionally, several studies pioneered by Reiman et al. have provided compelling evidence for FDG PET abnormalities in nondemented, as well as impaired ApoE $\varepsilon 4$ carriers vs. noncarriers [109-111]. For more information about PET findings related to $\mathrm{ApoE} \varepsilon 4$ status, please see the chapter by Nacmias et al. in this issue. More work is needed to establish how early FDG PET deficits become detectable in the course of the disease.

Nonetheless, published studies support the use of FDG PET in the early detection of $\mathrm{AD}$ and, thanks to the method's high sensitivity, regional hypometabolism in ADvulnerable brain regions has been proposed as an endophenotype of $\mathrm{AD}$. When a particular trait or biomarker typically found in conjunction with a disease is detected in persons who do not have the disease, the presence of that trait or biomarker is said to constitute an endophenotype [12]. While an endophenotype state does not mean that carriers will develop the full-blown disease, it indicates that they carry an increased risk compared with persons without the endophenotype [12]. FDG PET studies led to the identification of an AD endophenotype in still asymptomatic individuals at risk of developing the disease by virtue of having an AD-affected parent, especially those 
whose mothers had the disease. Evidence for a maternal link to LOAD further strengthens the notion that mitochondria-induced oxidative stress and metabolic impairments may be a primary promoter of neurodegeneration in at least some forms of LOAD, as reviewed below.

Detection of AD-related hypometabolism in asymptomatic individuals

Having a first-degree family history of LOAD is a major risk factor for developing the disease [112, 113]. However, the biological mechanisms through which a family history of $\mathrm{AD}$ confers risk to offspring are largely unknown. Recent FDG PET studies revealed that adult children of $\mathrm{AD}$-affected mothers are more likely to express an $\mathrm{AD}$ endophenotype than adult children of $\mathrm{AD}$-affected fathers. As parent gender effects on brain function had not been explored prior to publication of FDG PET reports, these findings set the stage for the exploration of a newly identified risk factor for $\mathrm{AD}$ (Fig. 4).

The first FDG PET study to show a "maternal effect" in AD used state-of-the-art full dynamic modeling with arterial input functions and showed that CMRglc was significantly lower in NL elderly with a maternal family history of $\mathrm{AD}(\mathrm{FHm})$ compared with those with a paternal family history (FHp) and with controls with a negative family history of AD (FH-) [114]. CMRglc reductions in FHm individuals involved the brain regions typically affected in clinical $\mathrm{AD}$ patients and ranged from $8 \%$ in frontal cortex to $27 \%$ in the PCC. No CMRglc differences were found between the FHp and FH- groups [114]. A subsequent 2-year longitudinal FDG PET study in NL individuals showed that CMRglc reductions over time are more severe in FHm than in FHp and FH- [115].

A further FDG PET study investigated whether CMRglc deficits in NL FHm are associated with advancing maternal age at birth [81]. Examination of a large sample of NL individuals with quantitative FDG PET scans showed that CMRglc in AD-vulnerable regions negatively correlated with maternal age at birth only in the FHm group [81]. In contrast, CMRglc was not influenced by maternal age at birth in FHp and FH- subjects. Advanced paternal age was not significantly associated with metabolism in any group [81]. It is known that maternal age has an impact on female reproductive capacity, possibly due to mitochondrial physiology. Oocyte quality is determined primarily by the

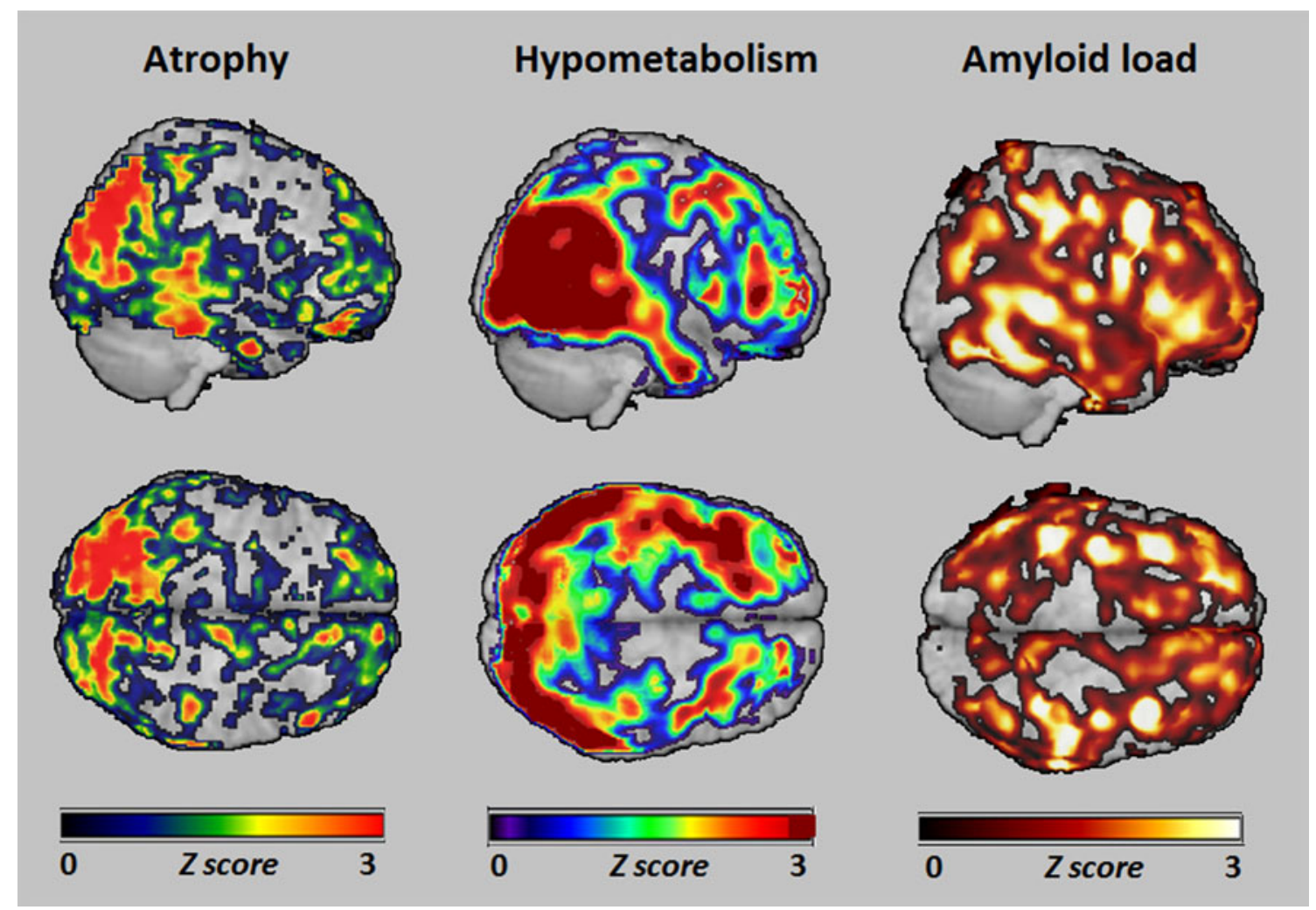

Fig. 4 Biomarker abnormalities in cognitively normal individuals with a maternal history of late-onset AD. From left to right, voxelbased detected patterns of gray matter volumes reductions on MRI (atrophy), reduced brain glucose metabolism on FDG PET and increased amyloid-beta load on PiB-PET in NL FHm compared to
FHp and FH- $[114,120,125,126]$. Statistical maps were obtained using voxel-based morphometry for MRI, and PET voxel-based analysis for FDG and PiB PET as implemented in Statistical Parametric Mapping (SPM) 
level of metabolism and, therefore, by mitochondrial physiology [116]. In general, a physiologically normal level of free radical production, when extended over a 30to 40-year period, could damage the oocyte mitochondrial DNA (mtDNA) sufficiently to reduce the oocytes' capacity for oxidative phosphorylation, explaining the "maternal age effect" [116]. Specifically for adult children of ADmothers, if maternal transmission of AD involves mtDNA, then affected mothers would have defective oocyte mitochondria since birth and throughout their lives, producing more oxidative stress than a non-affected individual. Giving birth at a later age would further extend the period of time in which these women's oocytes are exposed to large amounts of oxidative stress, which may lead to metabolic deficits in their offspring.

Importantly, in all above studies, no differences were observed between FHm males and females, or between ApoE $\varepsilon 4$ carriers and non-carriers [81, 114, 115]. Approximately $40 \%$ of all LOAD patients have at least one ApoE $\varepsilon 4$ allele [117]. Despite its well-established association with $\mathrm{AD}$, the ApoE $\varepsilon 4$ genotype has no clear familial pattern of transmission and appears to act as a risk modifier, by lowering the age at onset of clinical symptoms, rather than as a casual determinant [7]. CMRglc reductions were observed in NL FHm independent of their ApoE genotype, indicating that other factors contribute to the etiology and phenotypic expression of the disease in FHm. While LOAD does not follow classical Mendelian inheritance, the fact that $\mathrm{FHm}$ individuals have an increased risk of developing the disease suggests a genetic component. With all that is known about the genetic and molecular basis of glucose metabolism, FDG PET findings of selectively reduced metabolic activity in NL individuals with AD-affected mothers suggest involvement of mtDNA. As mentioned, mtDNA is exclusively maternally inherited in humans; it is transmitted equally to siblings, and mitochondrial genes are closely involved with glucose metabolism [14]. A defect in the ability to adequately consume glucose to produce energy, due to either inborn or pathology-induced mitochondrial abnormalities, may lead to early neuronal damage in predisposed individuals. Maternally transmitted mtDNA abnormalities, if confirmed, would act as an upstream event in NL individuals with AD-affected mothers, whereas mitochondrial damage secondary to AD pathology would be more likely in LOAD cases caused by factors other than mtDNA changes.

Several studies using biomarkers other than FDG PET have confirmed the association between maternal history of LOAD and presence of AD endophenotypes, in the form of reduced activity of platelet mitochondria COX (Complex IV of the mitochondrial ETC) [118], reduced $A \beta_{42 / 40}$ (reflecting increased sequestration in brain) and increased $\mathrm{F}_{2}$-isoprostanes (a marker of lipid membrane peroxidation produced by oxidative stress) in cerebrospinal fluid [119], reduced gray matter volumes on MRI (i.e., increased brain atrophy) [120-123], and increased retention of brain $N$ methyl- $\left[{ }^{11} \mathrm{C}\right] 2-\left(4^{\prime}\right.$-methylaminophenyl)-6-hydrox-

ybenzothiazol [Pittsburgh Compound-B, PiB [124], a marker of fibrillar $A \beta$ load [125]. On ${ }^{11} \mathrm{C}$-PiB PET, NL $\mathrm{FHm}$ showed increased and more widespread $\mathrm{PiB}$ retention compared with NL FHp and FH- [125]. Hypometabolism exceeded $\mathrm{A} \beta$ load in association with FHm status in NL and MCI populations [126, 127]. However, FHp subjects also displayed increased PiB retention in the PCC and medial frontal cortex, recording results intermediate between those of FHm and FH- [125-127]. Hypometabolism exceeded $A \beta$ accumulation may, therefore, be regarded as a common susceptibility feature for AD. However, other factors seem to be needed to trigger the "AD pathway" resulting in neuronal dysfunction and hypometabolism. Overall, these results indicate that NL FHm express a pathobiological phenotype characterized by $A \beta$-associated oxidative stress consistent with $\mathrm{AD}$, which might reflect an increased risk of developing the disease.

\section{Conclusions}

$\mathrm{AD}$ is characterized by progressive declines in memory, attention, and language. Often by the time patients express symptoms that warrant evaluation, too much irreversible brain damage has occurred for treatments to be effective. Therefore, it is of paramount importance to identify diagnostic markers, as well as disease mechanisms, for the preclinical stages of the disease when symptoms are not yet apparent. Changes in brain structure and function have been estimated to occur as many as 20-30 years before onset of symptoms in AD. Oxidative stress and increased ROS, which damage cell lipids and can eventually lead to neuronal death, are a natural byproduct of oxidative phosphorylation, which the brain relies on for energy production. As metabolic changes appear to be an early event in AD pathophysiology, FDG PET imaging is an invaluable tool in diagnosing $\mathrm{AD}$ thanks to the technique's capability to measure metabolic activity through regional glucose uptake and phosphorylation. Recent FDG PET findings have shown that adult children of AD-affected mothers are more likely to express an AD endophenotype than adult children with a paternal or negative family history of AD. As glucose metabolism is highly regulated by mitochondria and mtDNA is maternally inherited, impaired bioenergetics could be an upstream event in $\mathrm{AD}$ in these maternal cases. Identification of biomarker deficits in NL FHm and other at-risk populations would provide a unique opportunity for initiating $\mathrm{AD}$ therapies and general preventive interventions years, and possibly decades, prior 
to the onset of the clinical disease, presumably well before significant neuronal loss occurs. The combination of imaging and tailored genetics may help to clarify whether changes in the nuclear or mitochondrial genome and transcriptome account for pathogenic aging and AD.

Acknowledgments This study was supported by NIH/NIA grants AG035137, AG032554, AG13616 and the Alzheimer's Association IIRG-09-132030.

Conflict of interest Lisa Mosconi declares that she has no conflict of interest.

Human and Animal Studies This article does not contain any studies with human or animal subjects performed by the any of the authors.

\section{References}

1. Kukull WA, Higdon R, Bowen JD et al (2002) Dementia and Alzheimer disease incidence: a prospective cohort study. Arch Neurol 59:1737-1746

2. Braak H, Braak E (1991) Neuropathological stageing of Alzheimer-related changes. Acta Neuropathol 82:239-259

3. Delacourte A, David JP, Sergeant N et al (1999) The biochemical pathway of neurofibrillary degeneration in aging and Alzheimer's disease. Neurology 52:1158-1165

4. Price JL, Ko AI, Wade MJ, Tsou SK, McKeel DW, Morris JC (2001) Neuron number in the entorhinal cortex and CA1 in preclinical Alzheimer disease. Arch Neurol 58:1395-1402

5. Haass C, Selkoe DJ (2007) Soluble protein oligomers in neurodegeneration: lessons from the Alzheimer's amyloid betapeptide. Nat Rev Mol Cell Biol 8:101-112

6. Pratico D, Uryu K, Leight S, Trojanoswki JQ, Lee VM (2001) Increased lipid peroxidation precedes amyloid plaque formation in an animal model of Alzheimer amyloidosis. J Neurosci 21:4183-4187

7. Tanzi RE, Bertram L (2001) New frontiers in Alzheimer's disease genetics. Neuron 32:181-184

8. Selkoe DJ (2002) Alzheimer's disease is a synaptic failure. Science 298:789-791

9. Selkoe DJ (1997) Alzheimer's disease: genotypes, phenotypes, and treatments. Science 275:630-631

10. Blennow K, Hampel H, Weiner M, Zetterberg H (2010) Cerebrospinal fluid and plasma biomarkers in Alzheimer disease. Nat Rev Neurol. 6:131-144

11. Swerdlow RH, Burns JM, Khan SM (2010) The Alzheimer's disease mitochondrial cascade hypothesis. J Alzheimers Dis 20(Suppl 2):S265-S279

12. Swerdlow RH (2012) Mitochondria and cell bioenergetics: increasingly recognized components and a possible etiologic cause of Alzheimer's disease. Antioxid Redox Signal 16:1434-1455

13. Mattson MP, Magnus T (2006) Ageing and neuronal vulnerability. Nat Rev Neurosci 7:278-294

14. Lin MT, Beal MF (2006) Mitochondrial dysfunction and oxidative stress in neurodegenerative diseases. Nature 443:787-795

15. Beal MF (1995) Aging, energy, and oxidative stress in neurodegenerative diseases. Ann Neurol. 38:357-366

16. Ames BN, Shigenaga MK, Hagen TM (1993) Oxidants, antioxidants, and the degenerative diseases of aging. Proc Natl Acad Sci USA 90:7915-7922
17. Terry RD, Masliah E, Salmon DP et al (1991) Physical basis of cognitive alterations in Alzheimer's disease: synapse loss is the major correlate of cognitive impairment. Ann Neurol 30:572-580

18. Morrison JH, Hof PR (1997) Life and death of neurons in the aging brain. Science 278:412-419

19. Braak H, Braak E, Bohl J, Reintjes R (1996) Age, neurofibrillary changes, A beta-amyloid and the onset of Alzheimer's disease. Neurosci Lett 210:87-90

20. Sokoloff L (1977) Relation between physiological function and energy metabolism in the central nervous system. J Neurochem 29:13-26

21. Clarke DD, Sokoloff L (1994) Circulation and energy metabolism of the brain. In: Siegel GJ, Agranoff BW, Albers RW, Molinoff PB (eds) Basic Neurochemistry. Raven Press, New York, pp 645-680

22. Ray KK, Seshasai SR, Wijesuriya S et al (2009) Effect of intensive control of glucose on cardiovascular outcomes and death in patients with diabetes mellitus: a meta-analysis of randomised controlled trials. Lancet 373:1765-1772

23. Wong-Riley MT (1989) Cytochrome oxidase: an endogenous metabolic marker for neuronal activity. Trends Neurosci 12:94-101

24. Friede RI (1965) Enzyme histochemical studies of senile plaques. J Neuropathol Exp Neurol 24:477-491

25. Mecocci P, MacGarvey U, Beal MF (1994) Oxidative damage to mitochondrial DNA is increased in Alzheimer's disease. Ann Neurol. 36:747-751

26. Subbarao KV, Richardson JS, Ang LC (1990) Autopsy samples of Alzheimer's cortex show increased peroxidation in vitro. J Neurochem 55:342-345

27. Kish SJ, Bergeron C, Rajput A et al (1992) Brain cytochrome oxidase in Alzheimer's disease. J Neurochem 59:776-779

28. Cardoso SM, Proenca MT, Santos S, Santana I, Oliveira CR (2004) Cytochrome c oxidase is decreased in Alzheimer's disease platelets. Neurobiol Aging 25:105-110

29. Parker WD Jr, Mahr NJ, Filley CM et al (1994) Reduced platelet cytochrome c oxidase activity in Alzheimer's disease. Neurology 44:1086-1090

30. Valla J, Schneider L, Niedzielko T et al (2006) Impaired platelet mitochondrial activity in Alzheimer's disease and mild cognitive impairment. Mitochondrion 6:323-330

31. Gabuzda D, Busciglio J, Chen LB, Matsudaira P, Yankner BA (1994) Inhibition of energy metabolism alters the processing of amyloid precursor protein and induces a potentially amyloidogenic derivative. J Biol Chem 269:13623-13628

32. Reivich M, Kuhl D, Wolf A et al (1977) Measurement of local cerebral glucose metabolism in man with 18F-2-fluoro-2-deoxyD-glucose. Acta Neurol Scand Suppl 64:190-191

33. Sokoloff L, Reivich M, Kennedy C et al (1977) The [14C]deoxyglucose method for the measurement of local cerebral glucose utilization: theory, procedure, and normal values in the conscious and anesthetized albino rat. J Neurochem 28:897-916

34. Rocher AB, Chapon F, Blaizot X, Baron JC, Chavoix C (2003) Resting-state brain glucose utilization as measured by PET is directly related to regional synaptophysin levels: a study in baboons. Neuroimage. 20:1894-1898

35. Attwell D, Iadecola C (2002) The neural basis of functional brain imaging signals. Trends Neurosci 25:621-625

36. Mazziotta JC, Phelps ME (1986) Positron emission tomography studies of the brain. In: Phelps ME, Mazziotta JC, Schelbert H (eds) Positron emission tomography \& autoradiography: principles \& applications for the brain \& heart. Raven Press, New York, pp 493-579

37. Phelps ME, Huang SC, Hoffman EJ, Selin C, Sokoloff L, Kuhl DE (1979) Tomographic measurement of local cerebral glucose 
metabolic rate in humans with (F-18)2-fluoro-2-deoxy-D-glucose: validation of method. Ann Neurol. 6:371-388

38. Sokoloff L (1984) Modeling metabolic processes in the brain in vivo. Ann Neurol. 15(Suppl):S1-S11

39. Gjedde A, Wienhard K, Heiss WD et al (1985) Comparative regional analysis of 2-fluorodeoxyglucose and methylglucose uptake in brain of four stroke patients. With special reference to the regional estimation of the lumped constant. J Cereb Blood Flow Metab 5:163-178

40. Wienhard K, Pawlik G, Herholz K, Wagner R, Heiss WD (1985) Estimation of local cerebral glucose utilization by positron emission tomography of [18F]2-fluoro-2-deoxy-D-glucose: a critical appraisal of optimization procedures. J Cereb Blood Flow Metab 5:115-125

41. Hutchins GD, Holden JE, Koeppe RA, Halama JR, Gatley SJ, Nickles RJ (1984) Alternative approach to single-scan estimation of cerebral glucose metabolic rate using glucose analogs, with particular application to ischemia. J Cereb Blood Flow Metab 4:35-40

42. Lammertsma AA, Brooks DJ, Frackowiak RS et al (1987) Measurement of glucose utilisation with [18F]2-fluoro-2-deoxyD-glucose: a comparison of different analytical methods. J Cereb Blood Flow Metab 7:161-172

43. Patlak CS, Blasberg RG, Fenstermacher JD (1983) Graphical evaluation of blood-to-brain transfer constants from multipletime uptake data. J Cereb Blood Flow Metab 3:1-7

44. DiChiro G, Brooks RA (1988) PET quantitation: blessing and curse. J Nucl Med 29:1603-1604

45. Schmidt KC, Lucignani G, Sokoloff L (1996) Fluorine-18fluorodeoxyglucose PET to determine regional cerebral glucose utilization: a re-examination. J Nucl Med 37:394-399

46. Drzezga A, Grimmer T, Riemenschneider M et al (2005) Prediction of individual clinical outcome in MCI by means of genetic assessment and (18)F-FDG PET. J Nucl Med 46:1625-1632

47. Ishii K, Willoch F, Minoshima S et al (2001) Statistical brain mapping of 18F-FDG PET in Alzheimer's disease: validation of anatomic standardization for atrophied brains. J Nucl Med 42:548-557

48. Minoshima S, Frey KA, Koeppe RA, Foster NL, Kuhl DE (1995) A diagnostic approach in Alzheimer's disease using three-dimensional stereotactic surface projections of fluorine18-FDG PET. J Nucl Med 36:1238-1248

49. Minoshima S, Giordani B, Berent S, Frey KA, Foster NL, Kuhl DE (1997) Metabolic reduction in the posterior cingulate cortex in very early Alzheimer's disease. Ann Neurol 42:85-94

50. Mosconi L, Tsui WH, Herholz K et al (2008) Multicenter standardized 18F-FDG PET diagnosis of mild cognitive impairment, Alzheimer's disease, and other dementias. J Nucl Med 49:390-398

51. Herholz K, Salmon E, Perani D et al (2002) Discrimination between Alzheimer dementia and controls by automated analysis of multicenter FDG PET. Neuroimage. 17:302-316

52. Caroli A, Prestia A, Chen K et al (2012) Summary metrics to assess Alzheimer disease-related hypometabolic pattern with 18F-FDG PET: head-to-head comparison. J Nucl Med 53:592-600

53. Prestia A, Caroli A, van der Flier WM et al (2013) Prediction of dementia in MCI patients based on core diagnostic markers for Alzheimer disease. Neurology. 80:1048-1056

54. Anchisi D, Borroni B, Franceschi M et al (2005) Heterogeneity of brain glucose metabolism in mild cognitive impairment and clinical progression to Alzheimer disease. Arch Neurol 62:1728-1733

55. Signorini M, Paulesu E, Friston K et al (1999) Rapid assessment of regional cerebral metabolic abnormalities in single subjects with quantitative and nonquantitative [18F]FDG PET: A clinical validation of statistical parametric mapping. Neuroimage. 9:63-80

56. Teune LK, Bartels AL, de Jong BM et al (2010) Typical cerebral metabolic patterns in neurodegenerative brain diseases. Mov Disord 25:2395-2404

57. Yakushev I, Hammers A, Fellgiebel A et al (2009) SPM-based count normalization provides excellent discrimination of mild Alzheimer's disease and amnestic mild cognitive impairment from healthy aging. Neuroimage. 44:43-50

58. Wree A, Schleicher A (1988) The determination of the local cerebral glucose utilization with the 2-deoxyglucose method. Histochemistry 90:109-121

59. Hawkins RA, Mans AM, Davis DW, Hibbard LS, Lu DM (1983) Glucose availability to individual cerebral structures is correlated to glucose metabolism. J Neurochem 40:1013-1018

60. Iadecola C, Nakai M, Mraovitch S, Ruggiero DA, Tucker LW, Reis DJ (1983) Global increase in cerebral metabolism and blood flow produced by focal electrical stimulation of dorsal medullary reticular formation in rat. Brain Res 272:101-114

61. Baron JC, Lebrun-Grandie P, Collard P, Crouzel C, Mestelan G, Bousser MG (1982) Noninvasive measurement of blood flow, oxygen consumption, and glucose utilization in the same brain regions in man by positron emission tomography: concise communication. J Nucl Med 23:391-399

62. Raichle ME, Grubb RL Jr, Gado MH, Eichling JO, Ter-Pogossian MM (1976) Correlation between regional cerebral blood flow and oxidative metabolism. In vivo studies in man. Arch Neurol 33:523-526

63. Powers WJ, Perlmutter JS, Videen TO et al (1992) Blinded clinical evaluation of positron emission tomography for diagnosis of probable Alzheimer's disease. Neurology. 42:765-770

64. Fox PT, Raichle ME, Mintun MA, Dence C (1988) Nonoxidative glucose consumption during focal physiologic neural activity. Science 241:462-464

65. Sandman CA, O'Halloran JP, Isenhart R (1984) Is there an evoked vascular response? Science 224:1355-1357

66. Fox PT, Raichle ME (1986) Focal physiological uncoupling of cerebral blood flow and oxidative metabolism during somatosensory stimulation in human subjects. Proc Natl Acad Sci USA 83:1140-1144

67. Yarowsky P, Kadekaro M, Sokoloff L (1983) Frequencydependent activation of glucose utilization in the superior cervical ganglion by electrical stimulation of cervical sympathetic trunk. Proc Natl Acad Sci USA 80:4179-4183

68. Kadekaro M, Vance WH, Terrell ML, Gary H Jr, Eisenberg HM, Sokoloff L (1987) Effects of antidromic stimulation of the ventral root on glucose utilization in the ventral horn of the spinal cord in the rat. Proc Natl Acad Sci USA 84:5492-5495

69. Zeki S, Watson JD, Lueck CJ, Friston KJ, Kennard C, Frackowiak RS (1991) A direct demonstration of functional specialization in human visual cortex. J Neurosci 11:641-649

70. Petersen SE, Fox PT, Posner MI, Mintun M, Raichle ME (1988) Positron emission tomographic studies of the cortical anatomy of single-word processing. Nature 331:585-589

71. Chugani HT, Phelps ME, Mazziotta JC (1987) Positron emission tomography study of human brain functional development. Ann Neurol. 22:487-497

72. Phelps ME, Mazziotta JC (1985) Positron emission tomography: human brain function and biochemistry. Science 228:799-809

73. Schubert D (2005) Glucose metabolism and Alzheimer's disease. Ageing Res Rev. 4:240-257

74. Magistretti PJ, Pellerin L (1999) Astrocytes couple synaptic activity to glucose utilization in the brain. News Physiol Sci 14:177-182

75. Mosconi L (2005) Brain glucose metabolism in the early and specific diagnosis of Alzheimer's disease. FDG-PET studies in MCI and AD. Eur J Nucl Med Mol Imaging 32:486-510 
76. Silverman DH, Small GW, Chang CY et al (2001) Positron emission tomography in evaluation of dementia: regional brain metabolism and long-term outcome. JAMA 286:2120-2127

77. Herholz K (2010) Cerebral glucose metabolism in preclinical and prodromal Alzheimer's disease. Expert Rev Neurother 10:1667-1673

78. Caroli A, Testa C, Geroldi C et al (2007) Brain perfusion correlates of medial temporal lobe atrophy and white matter hyperintensities in mild cognitive impairment. J Neurol 254:1000-1008

79. de Leon MJ, Convit A, Wolf OT et al (2001) Prediction of cognitive decline in normal elderly subjects with $2-[(18) \mathrm{F}]$ fluoro-2-deoxy-D-glucose/poitron-emission tomography (FDG/ PET). Proc Natl Acad Sci USA 98:10966-10971

80. Mosconi L, De Santi S, Li J et al (2008) Hippocampal hypometabolism predicts cognitive decline from normal aging. Neurobiol Aging 29:676-692

81. Mosconi L, Tsui W, Murray J et al (2012) Maternal age affects brain metabolism in adult children of mothers affected by Alzheimer's disease. Neurobiol Aging 33:624. e1-e9

82. Sun FT, Schriber RA, Greenia JM, He J, Gitcho A, Jagust WJ (2007) Automated template-based PET region of interest analyses in the aging brain. Neuroimage 34:608-617

83. Mosconi L, De Santi S, Li Y et al (2006) Visual rating of medial temporal lobe metabolism in mild cognitive impairment and Alzheimer's disease using FDG-PET. Eur J Nucl Med Mol Imaging. 33:210-221

84. Drzezga A, Becker JA, Van Dijk KR et al (2011) Neuronal dysfunction and disconnection of cortical hubs in non-demented subjects with elevated amyloid burden. Brain. 134:1635-1646

85. Meguro K, Blaizot X, Kondoh Y, Le Mestric C, Baron JC, Chavoix C (1999) Neocortical and hippocampal glucose hypometabolism following neurotoxic lesions of the entorhinal and perirhinal cortices in the non-human primate as shown by PET. Implications for Alzheimer's disease. Brain. 122:1519-1531

86. Villain N, Desgranges B, Viader F et al (2008) Relationships between hippocampal atrophy, white matter disruption, and gray matter hypometabolism in Alzheimer's disease. J Neurosci 28:6174-6181

87. Morbelli S, Piccardo A, Villavecchia G et al (2010) Mapping brain morphological and functional conversion patterns in amnestic MCI: a voxel-based MRI and FDG-PET study. Eur J Nucl Med Mol Imaging 37:36-45

88. Mosconi L, Tsui WH, De Santi S et al (2005) Reduced hippocampal metabolism in MCI and AD: automated FDG-PET image analysis. Neurology 64:1860-1867

89. Loessner A, Alavi A, Lewandrowski KU, Mozley D, Souder E, Gur RE (1995) Regional cerebral function determined by FDGPET in healthy volunteers: normal patterns and changes with age. J Nucl Med 36:1141-1149

90. Moeller JR, Ishikawa T, Dhawan V et al (1996) The metabolic topography of normal aging. J Cereb Blood Flow Metab 16:385-398

91. De Santi S, de Leon MJ, Convit A et al (1995) Age-related changes in brain: II. Positron emission tomography of frontal and temporal lobe glucose metabolism in normal subjects. Psychiatr Q 66:357-370

92. Kalpouzos G, Chetelat G, Baron JC et al (2009) Voxel-based mapping of brain gray matter volume and glucose metabolism profiles in normal aging. Neurobiol Aging 30:112-124

93. Blass JP (2002) Alzheimer's disease and Alzheimer's dementia: distinct but overlapping entities. Neurobiol Aging 23:1077-1084

94. Grady CL, Haxby JV, Schlageter NL, Berg G, Rapoport SI (1986) Stability of metabolic and neuropsychological asymmetries in dementia of the Alzheimer type. Neurology 36:1390-1392

95. Haxby JV, Grady CL, Koss E et al (1990) Longitudinal study of cerebral metabolic asymmetries and associated neuropsychological patterns in early dementia of the Alzheimer type. Arch Neurol 47:753-760

96. Fouquet M, Desgranges B, Landeau B et al (2009) Longitudinal brain metabolic changes from amnestic mild cognitive impairment to Alzheimer's disease. Brain 132:2058-2067

97. Marshall GA, Monserratt L, Harwood D, Mandelkern M, Cummings JL, Sultzer DL (2007) Positron emission tomography metabolic correlates of apathy in Alzheimer disease. Arch Neurol 64:1015-1020

98. Kuczynski B, Reed B, Mungas D, Weiner M, Chui HC, Jagust W (2008) Cognitive and anatomic contributions of metabolic decline in Alzheimer disease and cerebrovascular disease. Arch Neurol 65:650-655

99. Woo BK, Harwood DG, Melrose RJ et al (2010) Executive deficits and regional brain metabolism in Alzheimer's disease. Int J Geriatr Psychiatry 25:1150-1158

100. Petersen RC, Roberts RO, Knopman DS et al (2009) Mild cognitive impairment: ten years later. Arch Neurol 66:1447-1455

101. Jagust W, Gitcho A, Sun F, Kuczynski B, Mungas D, Haan M (2006) Brain imaging evidence of preclinical Alzheimer's disease in normal aging. Ann Neurol 59:673-681

102. Mosconi L, Mistur R, Switalski R et al (2009) FDG-PET changes in brain glucose metabolism from normal cognition to pathologically verified Alzheimer's disease. Eur J Nucl Med Mol Imaging 36:811-822

103. Mosconi L, Sorbi S, de Leon MJ et al (2006) Hypometabolism exceeds atrophy in presymptomatic early-onset familial Alzheimer's disease. J Nucl Med 47:1778-1786

104. Nobili F, De Carli F, Frisoni GB et al (2009) SPECT predictors of cognitive decline and Alzheimer's disease in mild cognitive impairment. J Alzheimers Dis 17:761-772

105. Chetelat G, Desgranges B, de la Sayette V, Viader F, Eustache F, Baron JC (2003) Mild cognitive impairment: can FDG-PET predict who is to rapidly convert to Alzheimer's disease? Neurology. 60:1374-1377

106. Drzezga A, Lautenschlager N, Siebner H et al (2003) Cerebral metabolic changes accompanying conversion of mild cognitive impairment into Alzheimer's disease: a PET follow-up study. Eur J Nucl Med Mol Imaging 30:1104-1113

107. Valla J, Berndt JD, Gonzalez-Lima F (2001) Energy hypometabolism in posterior cingulate cortex of Alzheimer's patients: superficial laminar cytochrome oxidase associated with disease duration. J Neurosci 21:4923-4930

108. Liang WS, Reiman EM, Valla J et al (2008) Alzheimer's disease is associated with reduced expression of energy metabolism genes in posterior cingulate neurons. Proc Natl Acad Sci USA 105:4441-4446

109. Reiman EM, Caselli RJ, Chen K, Alexander GE, Bandy D, Frost J (2001) Declining brain activity in cognitively normal apolipoprotein E epsilon 4 heterozygotes: a foundation for using positron emission tomography to efficiently test treatments to prevent Alzheimer's disease. Proc Natl Acad Sci USA 98:3334-3339

110. Reiman EM, Chen K, Alexander GE et al (2004) Functional brain abnormalities in young adults at genetic risk for late-onset Alzheimer's dementia. Proc Natl Acad Sci USA 101:284-289

111. Reiman EM, Chen K, Alexander GE et al (2005) Correlations between apolipoprotein E epsilon4 gene dose and brain-imaging measurements of regional hypometabolism. Proc Natl Acad Sci USA 102:8299-8302

112. Farrer LA, O'Sullivan DM, Cupples LA, Growdon JH, Myers RH (1989) Assessment of genetic risk for Alzheimer's disease among first-degree relatives. Ann Neurol 25:485-493

113. Green RC, Cupples LA, Go R et al (2002) Risk of dementia among white and African American relatives of patients with Alzheimer disease. JAMA 287:329-336 
114. Mosconi L, Brys M, Switalski R et al (2007) Maternal family history of Alzheimer's disease predisposes to reduced brain glucose metabolism. Proc Natl Acad Sci USA 104:19067-19072

115. Mosconi L, Mistur R, Switalski R et al (2009) Declining brain glucose metabolism in normal individuals with a maternal history of Alzheimer disease. Neurology 72:513-520

116. Wilding M, Di Matteo L, Dale B (2005) The maternal age effect: a hypothesis based on oxidative phosphorylation. Zygote 13:317-323

117. Farrer LA, Cupples LA, Haines JL et al (1997) Effects of age, sex, and ethnicity on the association between apolipoprotein E genotype and Alzheimer disease. A meta-analysis. APOE and Alzheimer Disease Meta Analysis Consortium. JAMA 278:1349-1356

118. Mosconi L, de Leon M, Murray J et al (2011) Reduced mitochondria cytochrome oxidase activity in adult children of mothers with Alzheimer's disease. J Alzheimers Dis 27:483-490

119. Mosconi L, Glodzik L, Mistur R et al (2010) Oxidative stress and amyloid-beta pathology in normal individuals with a maternal history of Alzheimer's. Biol Psychiatry 68:913-921

120. Berti V, Mosconi L, Glodzik L et al (2011) Structural brain changes in normal individuals with a maternal history of Alzheimer's. Neurobiol Aging. 32:2325.e17-26

121. Honea RA, Swerdlow RH, Vidoni ED, Burns JM (2011) Progressive regional atrophy in normal adults with a maternal history of Alzheimer disease. Neurology 76:822-829
122. Honea RA, Swerdlow RH, Vidoni ED, Goodwin J, Burns JM (2010) Reduced gray matter volume in normal adults with a maternal family history of Alzheimer disease. Neurology $74: 113-120$

123. Andrawis JP, Hwang KS, Green AE et al (2012) Effects of ApoE4 and maternal history of dementia on hippocampal atrophy. Neurobiol Aging 33:856-866

124. Klunk WE, Engler H, Nordberg A et al (2004) Imaging brain amyloid in Alzheimer's disease with Pittsburgh Compound-B. Ann Neurol 55:306-319

125. Mosconi L, Rinne JO, Tsui WH et al (2010) Increased fibrillar amyloid-\{beta\} burden in normal individuals with a family history of late-onset Alzheimer's. Proc Natl Acad Sci USA 107:5949-5954

126. Mosconi L, Rinne JO, Tsui WH et al (2013) Amyloid and metabolic positron emission tomography imaging of cognitively normal adults with Alzheimer's parents. Neurobiol Aging 34:22-34

127. Mosconi L, Andrews R, Matthews DC (2013) Comparing brain amyloid deposition, glucose metabolism and atrophy in mild cognitive impairment with and without a family history of dementia. J Alzheimer's Dis 35:509-524 